\title{
Osteonecrosis of the Jaw in a Patient Treated with Denosumab and Temsirolimus
}

\author{
Nifosì $\mathrm{AF}^{1}$, Nifosì $\mathrm{L}^{2}$ and Nifosì $\mathrm{G}^{3 *}$ \\ ${ }^{1}$ Dentix Dental Clinic, Forli, Italy \\ ${ }^{2}$ European University of Valencia, Valencia, Spain \\ ${ }^{3}$ Department of Internal Medecine, Hemato-Oncology Clinic, Brugmann University Hospital Center, Brussels, Belgium
}

*Corresponding author: Nifosì G, Department of Internal Medecine, Hemato-Oncology Clinic, Brugmann University Hospital Center, Brussels, Belgium, Tel: 0032498502408, E-mail: g.nifosi@tin.it

Citation: Nifosì AF, Nifosì L, Nifosì G (2017) Osteonecrosis of the Jaw in a Patient Treated with Denosumab and Temsirolimus. SAJ Case Rep 4: 401

Article history: Received: 22 September 2017, Accepted: 28 November 2017, Published: 30 November 2017

\begin{abstract}
The number of osteonecrosis related medications of the jaw is constantly increasing, as is apparent from the many molecular target and immunological drugs in the last years introduced in the cancer treatment. In addition to bisphosphonates and denosumab, recently cases due to bevacizumab, sunitinib and ipilimumab have been described. The inhibition of the bone resorption and the angiogenesis block plays a fundamental role in the physiopathology of the osteonecrosis of the jaw. Also, the mammalian target of rapamycin pathway, through the reduced production of the hypoxia-inducible factors and the vascular endothelial growth factor, leads to the inhibition of angiogenesis. In this work, we report a case of ONJ during denosumab and temsirolimus treatment in a patient with metastatic renal cancer, in which temsirolimus may have played a role of contributory cause, synergizing the effect of the denosumab, in the development and maintenance of the bone necrosis.
\end{abstract}

Keywords: Osteonecrosis; Jaw; Denosumab; Angiogenesis; mTOR inhibitors

\section{Introduction}

The term osteonecrosis of the jaw (ONJ) was introduced by Marx in 2003 and a year later by Ruggiero [1,2]. Cases of spontaneous appearance have been described, even their frequency was still unknown [3]. ONJ is a rare treatment related side effect by antiresorptive drugs. At low doses, the bisphosphonates (BPs) osteoporosis treatment shows a very low risk (1:10,000-100,000), both high powerful doses for bone metastatic cancer patients gives the highest result (between 1 and 16\%) [4]. Regarding Denosumab (Dmab) on a monthly dose of $120 \mathrm{mg}$, a similar high risk was reported, pointing that its discontinuation might have been associated with more rapid ONJ resolution [5]. ONJ risk increased by concomitant local and systemic factors (Table 1). Recently, several cases by antiangiogenic and immunological drugs such as Sunitinib, Bevacizumab and Ipilimumab have been reported. The combination of Bps and this drugs result to have a really high risk. We present a case of ONJ in the course of the treatment by Denosumab and Temsirolimus.

\begin{tabular}{|c|c|}
\hline Local Factors & Systemical Factors \\
\hline Tooth extraction & $\begin{array}{c}\text { Metastatic cancer (breast, lung, } \\
\text { prostate, rein and myeloma) }\end{array}$ \\
\hline Dent alveolar surgery & Radiotherapy \\
\hline Periodontal disease & Chemotherapy \\
\hline Trauma & Chronic steroid treatment \\
\hline & Smoking \\
\hline & Anemia \\
\hline & Diabetes \\
\hline & Hypertension \\
\hline
\end{tabular}




\begin{tabular}{|c|c|}
\hline Local Factors & Systemical Factors \\
\hline & Hypothyroidism \\
\hline & Hyperparathyroidism \\
\hline & Renal failure \\
\hline
\end{tabular}

Table 1: Etiological factors of ONJ

\section{Case Report}

A 47 year-old-male non-smoker, caucasian patient, submitted three years earlier to laparoscopic radical nephrectomy for clear cell adenocarcinoma of the kidney, stage Ib, grade I, came under our observation for a small lung metastasis, multiple bone metastases and a high risk profile according to Motzer's criteria [6]. It was being treated with Temsirolimus (Tems), a mammalian target of rapamycin (mTOR) inhibitor, $25 \mathrm{mg}$ weekly and Denosumab (Dmab) $120 \mathrm{mg}$ monthly, with calcium and vitamin D supplementation. Before starting the Dmab all the necessary dental treatments were made (extractions and dental cleaning), according to the protocol $[7,8]$. After ten months of treatment, he complained jaw pain, inability to feed on, associated with inflammatory syndrome. The clinical examination highlights a bilateral osteonecrosis of the posterior mandible, grade II according to Ruggiero or grade IIa according to Bagan (Table 2 and 3). None dental predisposing factor was identified. The treatment started orally by giving him $1800 \mathrm{mg}$ clindamicine three times a day, $40 \mathrm{mg}$ morphine twice a day and locally $0.2 \%$ chlorhexidine rinses. Metastatic origin was excluded from the positron emission tomography negativity and therefore it was not considered to perform any biopsy. It was decided to discontinue Dmab, while Tems was continued, in view of the clinical improvement, characterized by the disappearance of the pulmonary nodule and a partial regression of the bone metastasis. One year after, the osteonecrosis of the mandible still persisted. The cone beam computed tomography showed a bilateral extension of the osteonecrosis with a bone cortical perforation (Figure 1). Tems was interrupted and slowly the osteonecrosis improved. The patient was treated with Nivolumab, an anti-PD-1 monoclonal antibody. After one year of treatment, the ONJ was completely resolved and the metastatic bone disease had a partial resolution (Figure 2).

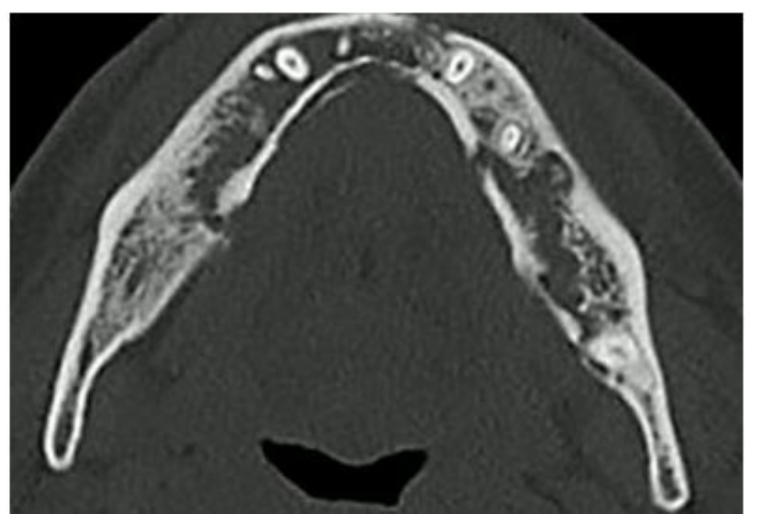

Figure 1: Cone beam computed tomography initial: bilateral osteonecrosis of the mandible with bone cortical perforation

\begin{tabular}{|c|c|}
\hline Stage 1 & Exposed necrotic bone with no infection and otherwise asymptomatic \\
\hline Stage 2 & Exposed necrotic bone with evidence of infection with or without purulent discharge \\
\hline Stage 3 & $\begin{array}{c}\text { Exposed necrotic bone with infection and extension radiographically to the inferior } \\
\text { border of the mandible or sinus floor in the maxilla or presence of an extra oral fistula } \\
\text { or pathologic fracture }\end{array}$ \\
\hline
\end{tabular}

Table 2: ONJ's staging (Ruggiero)

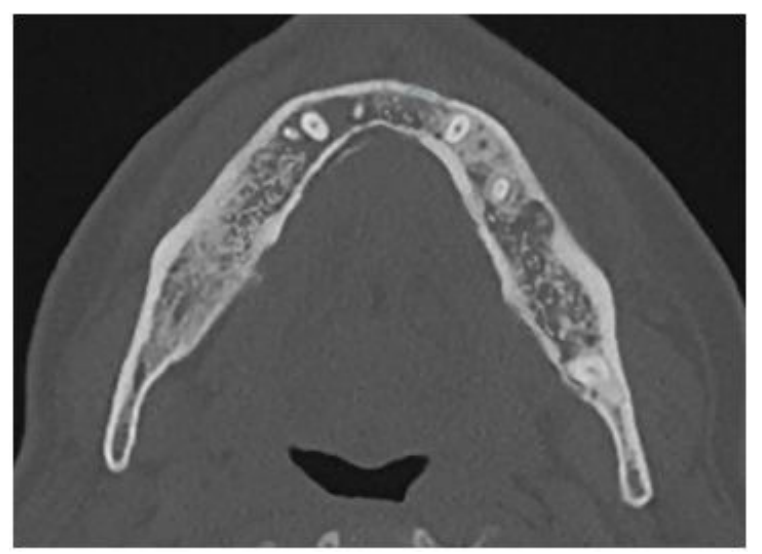

Figure 2: Cone beam computed tomography to one year: improvement of the osteonecrosis 


\begin{tabular}{|c|r|}
\hline Stage 1 & Exposed bone with no infection and otherwise asymptomatic \\
\hline Stage 2a & $\begin{array}{r}\text { Exposed necrotic bone, symptomatic, with evidence of soft tissues infection, that it } \\
\text { controls with conservative treatment and does not progressing }\end{array}$ \\
\hline Stage 2b & As a 2a, but that is not controlled by conservative treatment or progressing \\
\hline Stage 3 & As in the classification by Ruggiero \\
\hline
\end{tabular}

\section{Discussion}

The ONJ physiopathology is unclear. The posterior mandible region predilection is due to its density, its poor vascularization and to the continuous masticatory stimulus. The infections are important factors although it is not clear whether the infection proceeds or follow the necrosis. However, the bacterial presence stimulates the bone resorption and it may contribute to the bone necrosis. The vital bone is mixed irregularly with unvital bone and the bone surface is colonized by bacteria or Actinomyces colonies. The reduction of the bone turnover would play an important role and this explains the association of the ONJ with the antiresorptive drugs. The bone resorption is essential for the subsequent neoformation step, wich leads to the remodelling of this tissue. However, there are other low bone turnover conditions in which the ONJ problem does not arise. The BPs could act through a reduction of the mucosal blood supply, thanks to their antiangiogenic properties. In fact, ONJ has also been associated in some patients taking anti-angiogenic drugs [9]. Some experts suggest a genetic predisposition, such as farnesyl pyrophosphate synthase and cytochrome CYP450 CYP2C8 polymorphisms [10,11]. Dmab is a human monoclonal immunoglobulin (IgG2) that links the human receptor activator of the nuclear factor kappa-B ligand (RANKL) produced by osteoblast, preventing the activation of the RANK, receptor on the surface of the osteoclasts and the precursor cells. The reduction of the RANK-RANKL linking, consequently inhibits the osteoclasts formation, their activity and survival, besides the bone resorption is reduced and bone mass is enhanced [12]. Dmab has no anti-VEGF action. His half-life is roughly 26 days and not induce neutralising antibodies production. After administration of the first dose, the inhibition of the osteoclast activity occurs already after 6 hours, ending within 6 months after the last dose [13]. Consequently, unlike the BPs, Dmab does not accumulate at the bone level, whereby Dmab-related ONJ (DRONJ) is less intense that Bps-related ONJ (BRONJ), more rapidly reversible and it better responds to conservative treatment [14]. Tems is a Its inhibitor indicated for the high risk advanced renal cell carcinoma and relapsed or refractory mantle cell lymphoma treatment. The mTOR inhibition is the consequence of the binding of intracellular protein FKBP-12, which it follows a lower expression of D-type cyclins, c-myc and ornithine decarboxylase, regulatory proteins of the cell cycle, which results in the stop of the cell in G1 phase. It reduces also the phosphorylation of proteins (4E-BP 1 et $\mathrm{S} 6 \mathrm{~K}$ ) of the PI3 kinase/AKT thus blocking cell division. However, mTOR inhibition reduces hypoxia-inducible factors (HIF-1 and HIF-2 $\alpha$ ) and vascular endothelial growth factor (VEGF) production with tumoral angiogenesis block [15]. The mTOR pathway is also essential for both innate and adaptive immune responses. mTOR inhibition causes cell growth arrest and often immunosuppression [16]. The immunosuppressive state explains the infection susceptibility of the treated patients. Angiogenesis and ONJ have been linked in anti-angiogenic-related ONJ. Recently some cases of ONJ have been associated to bevacizumab, sunitinib and bisphosphonates $[17,18]$. Nowadays, the role of VEGF in the macrophage chemotaxis and osteoclast differentiation is well known, whereby is possible that the potent antiangiogenic activity of VEGF-targeting agents may inhibits the bone turnover and the host defenses, promoting the ONJ development and its severity. Besides, it is possible that these drugs and BPs may compromise the mucosal blood supply from the adjacent bone that result in epithelial breakdown [19]. In literature, there are reports of ONJ in patients receiving Everolimus without or with concomitant BPs and combination of Tems and Bevacizumab, but ONJ by Tems and Dmab is unknown [20-24]. We believe that the continuous Tems treatment caused micro vascular injury and reduced bone formation leading to more predispositions to infections. This explains the ONJ persistence. It remains to be assessed on a larger series if Tems alone can cause ONJ and if its association with antiresorptive drugs may have strengthening synergism in the osteonecrosis development.

\section{Learning Points:}

- mTOR inhibitors have antiproliferative, antiangiogenic and immunosuppressive effects.

- Temsirolimus can have a co-factor role in the development and maintenance of the DRONJ.

- First case of osteonecrosis of the jaw by denosumab and temsirolimus treatment.

- The oncologist should know the higher risk of ONJ in temsirolimus/denosumab treated patients and a close collaboration with the dental specialist, especially oral surgeon, is essential.

\section{References}

1. Marx RE (2003) Pamidronate (Aredia) and Zoledronate (Zometa) induced avascular necrosis of the jaw : a growing epidemic. J Oral Maxillofac Surg 61: 1115-7.

2. Ruggiero SL (2004) Osteonecrosis of the jaw associated with the use of bisphosphonates: a review of 63 cases. J Oral Maxillofac Surg 62: 527-34.

3. Reid IR, Cornish J (2012) Epidemiology and pathogenesis of osteonecrosis of the jaw. Nat Rev Rheumatol 8: 90-6.

4. Morrison A, Khan A, Tetradis S, Peters E (2015) Osteonecrosis of the jaw: an update for dentists. J Can Dent Assoc 81: f19.

5. O'Halloran M, Boyd NM, Smith A (2014) Denosumab and osteonecrosis of the jaws - the pharmacology, pathogenesis and a report of two cases. Aust Dent J 59: 516-9. 
6. Mekhail TM, Abou-Jawde RM, Boumerhi G, Malhi S, Wood L, et al. (2005) Validation and extension of the Memorial Sloan-Kattering prognostic factors model for survival in patients with previously untreated metastatic renal cell carcinoma. J Clin Oncol 23: 832-41.

7. Zhou H, Luo Y, Huang S (2010) Updates of mTOR inhibitors. Anticancer Agents Med Chem 10: 571-81.

8. Diniz-Freitos M, Limeres J (2016) Prevention of medication-related osteonecrosis of the jaw secondary to tooth extractions. A systematic review. Med Oral Pat Oral Cir Bucal 21: e250-9.

9. Christodoulou C, Pervena A, Klouvas G, Galani E, Falagas ME, et al. (2009) Combination of bisphosphonates and anti-angiogenic factors induced osteonecrosis of the jaw more frequently that bisphosphonates alone. Oncology 76: 209-11.

10. Xiao-Long Xu, Wen-Long Gou, Ali-Yuan W, Yu W, Quan-Yi G, et al. (2013) Basic research and clinical application of bisphosphonates in bone disease : what have we learned over the last 40 years ? J Transl Med 11: 303.

11. Sarasquete ME, Garcia-Sanz R, Marin L, Alcoceba M, Chillon MC, et al. (2008) Bisphosphonate-related osteonecrosis of the jaw is associated with polymorphisms of the cytochrome P450 CYP2C8 in multiple myeloma : a genome wide single nucleotide polymorphism analysis. Blood 112: 2709-12.

12. De Oliveira CC, Cavalcante-Brizeno LA, de Sousa FB, Mota MR, Alves AP (2016). Osteonecrosis of the jaw by receptor activator of nuclear factor-kappa B ligand (Denosumab) - Review. Med Oral Patol Oral Cir Bucal 21: e431-9.

13. Sohn W, Simiens MA, Jaeger K, Hutton S, Jang G (2014) The pharmacokinetics and pharmacodynamics of denosumab in patients with advanced solid tumours and bone metastases : a systematc reviw. Br J Clin Pharmacology 78: 477-87.

14. Favia G, Tempesta A, Limongelli L, Crincoli V, Maiorano E (2016) Medication-related osteonecrosis of the jaw: considerations on a new antiresorptive therapy (denosumab) and treatment out come after a 13-year experience. Int J Dent 1801676.

15. Coutte L, Dreyer C, Sablin MP, Faivre S, Raymond E (2012) PI3K-AKT-mTOR pathway and cancer. Bull Cancer 99: 173-80.

16. Cobbold SP (2013) The mTOR pathway and integrating immune regulation. Immunology 140: 391-8.

17. Estilo CL, Fornier M, Farooki A, Carlson D, Bohle G, et al. (2008) Osteonecrosis of the jaw related to bevacizumab. J Clin Oncol $20: 4037$.

18. Hoefert S, Eufinger H (2010) Sunitinib may raise the risk of bisphosphonate-related osteonecrosis of the jaw : presentation of three cases. Oral Surg Oral Med Oral Pathol Oral Radiol 110: 463-9.

19. Cornish J, Bava U, Callon KE, Bai J, Naot D, et al. (2011) Bone-bound bisphosphonate inhibits growth of adjacent non-bone cells. Bone 49: 710-6.

20. Yamamoto D, Tsubota Y, Utsunomiya T, Sueoka N, Ueda A et al. (2017) Osteonecrosis of the jaw associated with everolimus : A case report. Mol Clin Oncol 6: 255-7.

21. Giancola F, Campisi G, Lo Russo L, Lo Muzio L, Di Fede O, et al. (2013) Osteonecrosis of the jaw related to everolimus and bisphosphonate: a unique case report? Ann Stomatol (Roma) suppl 2: 20-1.

22. Martini V, Bonacina R, Mariani (2014) Osteonecrosis of the jaw in a patient treated with zoledronic acid and everolimus: a case report. Ann Stomatol 5.2: 26.

23. Kim DW, Jung YS, Park HS, Jung HD (2013) Osteonecrosis of the jaw related to everolimus : A case report. Br J Oral Maxillofac Surg 51: e302-4.

24. Santos-Silva AR, Belizário Rosa GA, Castro GD, Dias RB, Prado Ribeiro AC, et al. (2013) Osteonecrosis of the mandible associated with bevacizumab therapy. Oral Surg Oral Med Oral Pathol Oral Radiol 115: e32-6. 